\title{
Single dose bevacizumab as treatment for symptomatic, corticosteroid-refractory pseudoprogression after chemoradiation therapy for newly diagnosed glioblastoma: A case report
}

\author{
Taylor M. Morris ${ }^{1}$, Isabelle Vallieres ${ }^{2}$, Andrew Attwell ${ }^{3}$, R Petter Tonseth ${ }^{4}$, Natella Jafarova ${ }^{3}$ and David L. Saltman $^{3 *}$ \\ ${ }^{1}$ Louisiana State University School of Medicine, USA \\ ${ }^{2}$ Department Radiation Oncology, British Columbia Cancer Agency, Canada \\ ${ }^{3}$ Department Medical Oncology, British Columbia Cancer Agency, Canada \\ ${ }^{4}$ Department of Functional Imaging, BC Cancer Agency, Canada
}

\begin{abstract}
The survival of patients with glioblastoma has improved significantly since the introduction of radiation therapy with concurrent and adjuvant temolozolomide as standard therapy. However, symptomatic increases in MRI enhancement and edema mimicking tumor progression (pseudoprogression) have been well described after dual modality chemoradiation. We report the outcome of a glioblastoma patient after the administration of a single dose of bevacizumab for corticosteroidrefractory pseudoprogression. A 60-year old male with an unresectable glioblastoma developed symptoms and MRI changes suggestive of either progression or pseudoprogression during cycle one of adjuvant temozolomide, six weeks after completing treatment with radiation therapy and concomitant temozolomide. The patient's condition's did not respond adequately to high doses of corticosteroids but improved rapidly after a single dose of bevacizumab. MRI taken two weeks after the administration of bevacizumab showed significant improvement. The patient received no further therapy or corticosteroids. Follow-up MRI three months later showed no evidence of progression. We report the case of a glioblastoma patient suspected of developing pseudoprogression after combined modality chemoradiation. The dramatic symptomatic improvement after a single dose of bevacizumab suggests that a short course of the drug may be an effective strategy for the treatment of pseudoprogression in patients who respond poorly to corticosteroids.
\end{abstract}

\begin{abstract}
Abbreviations: RT: Radiation Therapy, TMZ: Temozolomide, FLAIR: Fluid Attenuation Inversion Recovery, MRI: Magnetic Resonance Imaging, IDH1: Isocitrate Dehydrogenase 1, MGMT: O6-Methylguanine-DNA-mehtyltransferase, LMWH: Low Molecular Weight Heparin, VEGF: Vascular Endothelial Growth Factor, BNCT: Boron Neutron Capture Therapy, VTE: Venous Thromboembolism, RN: Radiation Necrosis
\end{abstract}

\section{Introduction}

Glioblastoma is the most common primary malignant brain neoplasm in adults. The standard of care at diagnosis for glioblastoma is surgical resection, when possible, followed by radiation therapy (RT) and concomitant and adjuvant temozolomide (TMZ) chemotherapy. This treatment approach has been shown to prolong both progressionfree and overall survival of these patients [1].

Pseudoprogression is an early-to-subacute post-treatment effect observed on the imaging of high-grade gliomas usually occurring within the initial three months following the start of chemoradiation therapy [2-6]. The increased gadolinium contrast enhancement on magnetic resonance imaging (MRI) can be misinterpreted as tumor recurrence or radiation necrosis $(\mathrm{RN})$. Radiation therapy with concurrent TMZ chemotherapy followed by adjuvant TMZ is associated with the occurrence of pseudoprogression in up to $38 \%$ (28\% to $66 \%)$ of cases [7]. The incidence of pseudoprogression appears to be higher in malignant gliomas with O6-Methylguanine-DNA-mehtyltransferase (MGMT) gene promoter methylation, which is associated with improved treatment outcomes [8]. While the optimal approach for the management of symptomatic pseudoprogression has not yet been determined, most patients are treated empirically with corticosteroids. Here we present a case of pseudoprogression with severe neurologic symptoms refractory to corticosteroids that resolved fully following the administration of only a single dose of bevacizumab.

\section{Methods and case presentation}

A 60-year old male with no significant past medical history and no family history of cancer presented in November 2014 with severe headaches. An MRI of the brain showed a $4.5 \mathrm{~cm}$ enhancing mass centered in the right temporal lobe and extending into the lateral aspect of the splenium of the corpus callosum (Figure 1A and 1E). The mass was deemed unresectable because of its location. A biopsy revealed a

Correspondence to: David L. Saltman, Department Medical Oncology, British Columbia Cancer Agency, 2410 Lee Avenue, Victoria, British Columbia, V8R 6V5, Canada; E-mail: David.saltman@bccancer.bc.ca

Key words: glioblastoma, pseudoprogression, radiation therapy, temozolomide, bevacizumab; O6-methylguanine-DNA methyltransferase (MGMT)

Received: July 27, 2015; Accepted: August 20, 2015; Published: August 24, 2015 
Morris TM (2015) Single dose bevacizumab as treatment for symptomatic, corticosteroid-refractory pseudoprogression after chemoradiation therapy for newly diagnosed glioblastoma: A case report

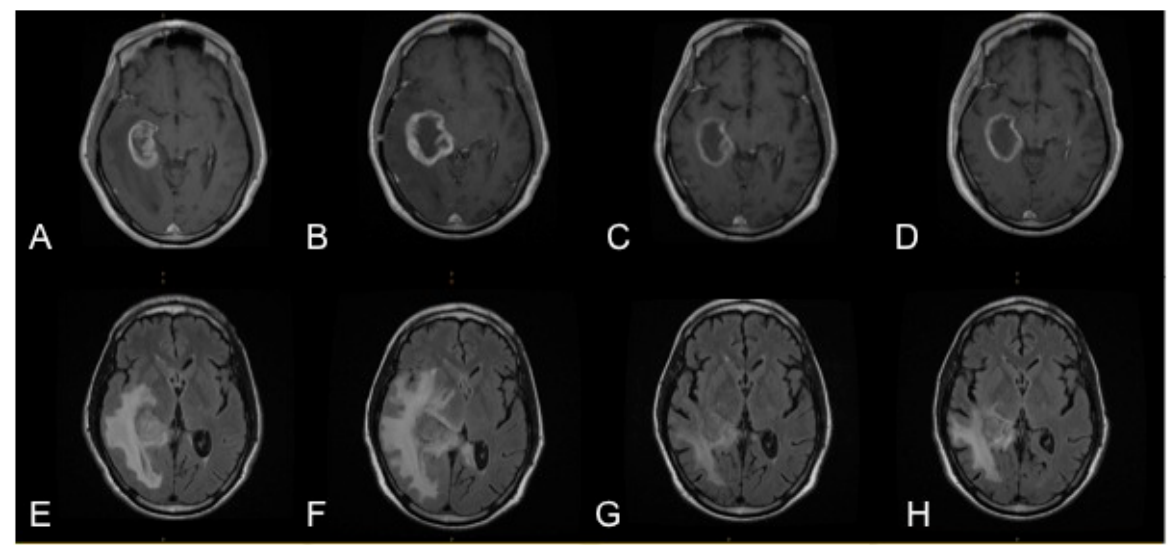

Figure 1. MRI of brain before chemoradiation, gadolinium-enhanced on T1-weighed image (A), and FLAIR image (E). MRI six weeks after chemoradiation showing increased contrast enhancement, edema and midline shift (B and F). Images taken 3 weeks after the administration of a single dose of bevacizumab demonstrating the marked decrease in contrast enhancement and edema (C and $\mathrm{G})$, and at first follow-up 12 weeks later (D and $\mathrm{H})$ showing stable disease.

glioblastoma. Immunostaining demonstrated the tumor to be IDH1 mutation negative and 533 positive. The MGMT gene was methylated.

The patient was treated with $60 \mathrm{~Gy}$ of RT in 30 fractions to the right temporal lobe together with concomitant TMZ $75 \mathrm{mg} / \mathrm{m}^{2}$ daily. Dexamethasone, which was started at diagnosis, was tapered during his treatment to $1 \mathrm{mg}$ daily. Three weeks after starting the first cycle of adjuvant temozolomide at a dose of $150 \mathrm{mg} / \mathrm{m}^{2}$ daily for five days of a monthly cycle, the patient developed headaches and nausea. $\mathrm{He}$ was advised to increase the dose of his dexamethasone but had a grand mal seizure and was admitted to hospital. MRI of the brain showed an increase in the size of the right temporal lobe mass with extension along the corpus callosum (Figure 1B,1F). There was extensive midline shift and transtentorial herniation with mass effects on the brainstem and right lateral ventricle. The patient received $16 \mathrm{mg}$ of dexamethasone and phenytoin with some improvement in his symptoms and no further seizures. He was started on a prophylactic dose of low molecular weight heparin (LMWH) to reduce the risk of venous thromboembolism (VTE). However, attempts at reducing the dose of steroids resulted in worsening symptoms, which did not respond to higher doses of dexamethasone. Two weeks after admission the patient was given $10 \mathrm{mg} / \mathrm{kg}$ of bevacizumab intravenously. Two days after receiving bevacizumab, the patient developed severe chest pain with an elevated troponin but normal electrocardiogram. A CT of the chest showed bilateral pulmonary emboli. The patient was switched to full-dose LMWH. The family requested that hospice be consulted and that no future active cancer therapy be given. The dexamethasone was rapidly tapered and stopped but the patient's neurological condition improved dramatically within days. MRI of the brain 10 days after bevacizumab showed a decrease in the size of the right temporal lobe mass and a significant reduction in the enhancement and mass effect (Figure 1C and $1 \mathrm{G}$ ). The patient was discharged home 5 weeks after his admission on phenytoin but without corticosteroids. No further chemotherapy was given. A follow-up visit 4 months after the administration of bevacizumab revealed no MRI or symptomatic evidence of progression (Figure 1D and 1H).

\section{Discussion}

As a result of the introduction RT with concomitant and adjuvant TMZ as treatment of choice for newly diagnosed glioblastoma following surgery, certain clinical response patterns and associated imaging findings are now being observed with increased frequency making the assessment of tumor response to therapy challenging. Contrast enhancement in brain tumors post-treatment is nonspecific and may not always be indicative of tumor response. Both pseudoprogression, an increase in the non-tumoral enhancing area, and pseudoresponse, a decrease in the enhancing area, show that enhancement alone is not a reliable measure of tumor activity but rather reflects a disturbed bloodbrain barrier [4,9].

Changes in contrast enhancement detected by MRI during or shortly after treatment can mimic early tumor progression. Increased enhancement can be caused by a variety of non-tumoral processes including treatment-related inflammation, post-surgical changes, ischemia, subacute radiation effects, and radiation necrosis [10-12].

Pseudoprogression is best diagnosed through serial MRIs because no established method of imaging is yet capable of providing a definitive diagnosis of true tumor progression versus enhancement changes due to other reasons [2-7]. Increased contrast enhancement and peritumoral edema that decrease with time are characteristic of pseudoprogression whereas such changes are stable with bona fide tumor progression.

After completion of concurrent RT and TMZ (most commonly within the first 3 months after completing treatment, but with a range in occurrence from the first few weeks to 6 months post-treatment), patients with high-grade brain tumors can present with an increase in contrast-enhancing lesion size, followed often by subsequent improvement or stabilization without further treatment [2-7]. This subacute treatment-related reaction can occur with or without clinical deterioration, but pseudoprogression is clinically asymptomatic in most patients [13].

Pseudoprogression can occur following RT alone but is far more frequently seen subsequent to the adoption of concomitant RT and TMZ therapy as the standard of care for newly diagnosed high-grade gliomas. Pseudoprogression can also occur following other chemotherapy regimens, including subsequent to placement of chemotherapy-infused wafers in the surgical cavity, and has been described following boron neutron capture therapy (BNCT) for recurrent malignant gliomas as well [2-6,14].

Pseudoprogression is thought to be induced by a pronounced 
Morris TM (2015) Single dose bevacizumab as treatment for symptomatic, corticosteroid-refractory pseudoprogression after chemoradiation therapy for newly diagnosed glioblastoma: A case report

local tissue reaction with an inflammatory component, edema, and abnormal vessel permeability, resulting in new or increased contrast enhancement on MRI examinations. It may represent at least in part an active inflammatory response against the tumor, and some studies have found an association between the incidence of pseudoprogression and increased survival [2-7,9].

The MGMT gene promoter methylation status is a known prognostic factor in patients with glioblastoma with tumors deficient in MGMT expression (i.e., with MGMT promoter methylation) exhibiting increased sensitivity to TMZ. As a consequence, patients with low MGMT expression benefit more from adjuvant TMZ and have an increased overall survival. Of note, patients with methylated MGMT experience pseudoprogression more frequently than those with $M G M T$ promoter hypo-methylation and gene expression, presumably at least in part due to the higher tumor sensitivity to treatment $[8,15]$. Pseudoprogression as seen on imaging may occur in up to 91\% of glioblastoma patients with methylated MGMT. By contrast, approximately $60 \%$ of patients with GBM possessing unmethylated $M G M T$ and gene expression have true early tumor progression [8]. Thus, MGMT gene methylation status may be an important factor to assist in the evaluation of imaging changes early in the post-RT treatment phase. Other tumor characteristics that may be of assistance in evaluating imaging changes in GBM post-therapy may also exist. Overexpression of the $\mathrm{p} 53$ protein (as in our patient), has been reported to positively correlate with the development of pseudoprogression [16], although, confirmation of this finding awaits independent study.

In general, both tumor progression and pseudoprogression should be considered possibilities for focal enhancement appearing in the irradiated area within 6 months after completion of RT. The differentiation of a recurrent/progressive tumor from radiation injury is often a clinical dilemma, given that pseudoprogression may influence decisions to continue adjuvant chemotherapy rather than changing to a second-line therapy for recurrent disease [27,17]. If a post-chemoradiotherapy follow-up MRI examination demonstrates complete or partial response or stable disease (i.e., smaller or stable tumor enhancement), maintenance of chemotherapy is typically continued. However, when enlargement occurs, subsequent management steps can be uncertain. If pseudoprogression is suspected, perhaps based on $M G M T$ status and/or very early changes in imaging during the first months post-treatment, ongoing chemotherapy with temozolomide might is continued with close monitoring. Adjuvant TMZ was not continued in our patient after the resolution of the pseudoprogression because of the severity of the symptoms and at the request of the patient.

In clinically symptomatic patients, as with the case presented here, more options for treatment of pseudoprogression must be considered including cessation of therapy and corticosteroid therapy. In those patients who experience clinical deterioration despite escalating doses of corticosteroids, additional intervention is warranted. Bevacizumab, an anti-vascular endothelial growth factor (VEGF) antibody, has been used for the treatment of symptomatic RN [18-20]. Given that it is difficult to definitively distinguish certain features of RN from pseudoprogression, Miyatake and colleagues were prompted to treat two patients with clinically symptomatic pseudoprogression following BNCT for recurrent tumors using intravenous bevacizumab - in both cases with rapid and marked improvement in neuroimaging abnormalities and symptoms [14]. In contrast to our patient, who was administered only a single intravenous dose of bevacizumab with beneficial effect, the two patients described by Miyatake et al. were treated with $5 \mathrm{mg} / \mathrm{kg}$ bevacizumab intravenous biweekly for 6 cycles. Since the clinical experience with treatment of symptomatic pseudoprogression using bevacizumab is still quite limited, the optimal dosing regimen remains to be established. Regardless, our experience suggests that minimal exposure to bevacizumab - even a single dose - may be adequate for the resolution of pseudoprogression-associated symptoms. Controlled studies that address this topic are warranted, both for optimal patient care and to ensure the use of bevacizumab in the most economical manner possible.

Miyatake et al. reported the ability to decrease the corticosteroid dose in their patients with symptomatic pseudoprogression after bevacizumab therapy [14]. Our patient's dexamethasone dose was tapered and discontinued rapidly over a week after a decision was made to stop all active treatment, yet his condition improved dramatically suggesting the pivotal role of the single dose of bevacizumab.

The basis for the efficacy of bevacizumab is consistent with the biology underlying the pathogenesis of symptomatic pseudoprogression and of radiation necrosis. There is no obvious histological difference between $\mathrm{RN}$ and pseudoprogression, with necrosis being the central feature of both [21,22]. In addition, $\mathrm{RN}$ is also characterized by marked enhancement in the tumor bed but without actual tumor, like pseudoprogression. It may not be incorrect to consider pseudoprogression a mild and self-limiting variant of treatment-related necrosis [2-7]. Prominent angiogenesis is common at the boundary of central necrosis and normal brain tissue in both entities. Clinically, pseudoprogression usually occurs at a relatively early stage after intensive treatments and is self-limited in most cases without treatment. In contrast, $\mathrm{RN}$ often shows severe symptoms and occurs at least 6 months following radiotherapy, can be long lasting, and improves only with intensive therapy such as surgery or bevacizumab administration $[23,24]$. Human surgical specimens of RN have been shown to exhibit overproduction of VEGF in reactive astrocytes in the perinecrotic area, resulting in leaky angiogenesis with perifocal edema [22]. Bevacizumab would serve to neutralize this overproduced VEGF and subsequently reduce the associated edema $[19,20]$.

\section{Conclusions}

In summary, we describe a glioblastoma patient with symptomatic pseudoprogression refractory to corticosteroids whose symptoms and MRI appearance improved significantly after only a single dose of intravenous bevacizumab. While bevacizumab has been utilized in the treatment of symptomatic radiation necrosis with good efficacy, there are very few descriptions in the literature of its use for symptomatic pseudoprogression. Our observation may be informative in the management of other patients with pseudoprogression; however, additional studies to both confirm the efficacy and safety of bevacizumab in this clinical setting are warranted.

\section{Consent}

Written informed consent was obtained from the patient for publication of this case report and any accompanying images. A copy of the written consent is available for review from the Editor of this journal.

\section{Authors contributions}

TMM and NJ reviewed the clinical data and drafted and critically reviewed the manuscript. RPT reviewed the imaging studies and help draft the manuscript. IV, AA and DS treated the patient, reviewed the 
Morris TM (2015) Single dose bevacizumab as treatment for symptomatic, corticosteroid-refractory pseudoprogression after chemoradiation therapy for newly diagnosed glioblastoma: A case report

data and drafted the manuscript. All authors read and approved the final manuscript.

\section{References}

1. Stupp R, Mason WP, van den Bent MJ, Weller M, Fisher B, et al. (2005) Radiotherapy plus concomitant and adjuvant temozolomide for glioblastoma. $N$ Engl J Med 352: 987-996. [Crossref]

2. Brandsma D, Stalpers L, Taal W, Sminia P, van den Bent MJ (2008) Clinical features, mechanisms, and management of pseudoprogression in malignant gliomas. Lancet Oncol 9: 453-461. [Crossref]

3. Chaskis C, Neyns B, Michotte A, De Ridder M, Everaert H (2009) Pseudoprogression after radiotherapy with concurrent temozolomide for high-grade glioma: clinical observations and working recommendations. Surg Neurol 72: 423-428. [Crossref]

4. Hygino da Cruz LC Jr, Rodriguez I, Domingues RC, Gasparetto EL, Sorensen AG (2011) Pseudoprogression and pseudoresponse: imaging challenges in the assessment of posttreatment glioma. AJNR Am J Neuroradiol 32: 1978-1985. [Crossref]

5. Knudsen-Baas KM, Moen G, Fluge Ø, Storstein A (2013) Pseudoprogression in highgrade glioma. Acta Neurol Scand: 31-37. [Crossref]

6. Kruser TJ, Mehta MP, Robins HI (2013) Pseudoprogression after glioma therapy: a comprehensive review. Expert Rev Neurother 13: 389-403. [Crossref]

7. Fink J, Born D, Chamberlain MC (2011) Pseudoprogression: relevance with respect to treatment of high-grade gliomas. Curr Treat Options Oncol 12: 240-252. [Crossref]

8. Brandes AA, Franceschi E, Tosoni A, Blatt V, Pession A, et al. (2008) MGMT promoter methylation status can predict the incidence and outcome of pseudoprogression after concomitant radiochemotherapy in newly diagnosed glioblastoma patients. J Clin Oncol 26: 2192-2197. [Crossref]

9. Brandsma D, van den Bent MJ (2009) Pseudoprogression and pseudoresponse in the treatment of gliomas. Curr Opin Neurol 22: 633-638. [Crossref]

10. Kumar AJ, Leeds NE, Fuller GN, Van Tassel P, Maor MH, et al. (2000) Malignan gliomas: MR imaging spectrum of radiation therapy- and chemotherapy-induced necrosis of the brain after treatment. Radiology 217: 377-384. [Crossref]

11. Ulmer S, Braga TA, Barker FG 2nd, Lev MH, Gonzalez RG, et al. (2006) Clinical and radiographic features of peritumoral infarction following resection of glioblastoma. Neurology 67: 1668-1670. [Crossref]

12. de Wit MC, de Bruin HG, Eijkenboom W, Sillevis Smitt PA, van den Bent MJ (2004) Immediate post-radiotherapy changes in malignant glioma can mimic tumor progression. Neurology 63: 535-537. [Crossref]
13. Taal W, Brandsma D, de Bruin HG, Bromberg JE, Swaak-Kragten AT, et al. (2008) Incidence of early pseudo-progression in a cohort of malignant glioma patients treated with chemoirradiation with temozolomide. Cancer 113: 405-410. [Crossref]

14. Miyatake S, Furuse M, Kawabata S, Maruyama T, Kumabe T, et al. (2013) Bevacizumab treatment of symptomatic pseudoprogression after boron neutron capture therapy for recurrent malignant gliomas. Report of 2 cases. Neuro Oncol 15: 650-655. [Crossref]

15. Brandes AA, Tosoni A, Franceschi E, Sotti G, Frezza G, et al. (2009) Recurrence pattern after temozolomide concomitant with and adjuvant to radiotherapy in newly diagnosed patients with glioblastoma: correlation with MGMT promoter methylation status. J Clin Oncol 27: 1275-1279. [Crossref]

16. Kang HC, Kim CY, Han JH, Choe GY, Kim JH, et al. (2011) Pseudoprogression in patients with malignant gliomas treated with concurrent temozolomide and radiotherapy: potential role of p53. J Neurooncol 102: 157-162. [Crossref]

17. Sanghera P, Perry J, Sahgal A, Symons S, Aviv R, et al. (2010) Pseudoprogression following chemoradiotherapy for glioblastoma multiforme. Can J Neurol Sci 37: 3642. [Crossref]

18. Wang Y, Pan L, Sheng X, Mao Y, Yao Y, et al. (2012) Reversal of cerebral radiation necrosis with bevacizumab treatment in 17 Chinese patients. Eur J Med Res 17: 25. [Crossref]

19. Furuse M, Kawabata S, Kuroiwa T, Miyatake S (2011) Repeated treatments with bevacizumab for recurrent radiation necrosis in patients with malignant brain tumors: a report of 2 cases. J Neurooncol 102: 471-475. [Crossref]

20. Levin VA, Bidaut L, Hou P, Kumar AJ, Wefel JS, et al. (2011) Randomized doubleblind placebo-controlled trial of bevacizumab therapy for radiation necrosis of the central nervous system. Int J Radiat Oncol Biol Phys 79: 1487-1495. [Crossref]

21. Miyatake S, Kawabata S, Nonoguchi N, Yokoyama K, Kuroiwa T, et al. (2009) Pseudoprogression in boron neutron capture therapy for malignant gliomas and meningiomas. Neuro Oncol 11: 430-436. [Crossref]

22. Nonoguchi N, Miyatake S, Fukumoto M, Furuse M, Hiramatsu R, et al. (2011) The distribution of vascular endothelial growth factor-producing cells in clinical radiation necrosis of the brain: pathological consideration of their potential roles. $J$ Neurooncol 105: 423-431. [Crossref]

23. Siu A, Wind JJ, Iorgulescu JB, Chan TA, Yamada Y, et al. (2012) Radiation necrosis following treatment of high grade glioma--a review of the literature and current understanding. Acta Neurochir (Wien) 154: 191-201. [Crossref]

24. Parvez K, Parvez A, Zadeh G (2014) The diagnosis and treatment of pseudoprogression, radiation necrosis and brain tumor recurrence. Int J Mol Sci 15: 11832-11846. [Crossref]

Copyright: (C) 2015 Morris TM. This is an open-access article distributed under the terms of the Creative Commons Attribution License, which permits unrestricted use, distribution, and reproduction in any medium, provided the original author and source are credited. 